THE ROLE OF WAVES ON MIXING IN SHALLOW WATERS

\begin{tabular}{|r|l|}
\hline Journal: & Canadian Journal of Civil Engineering \\
\hline Manuscript ID & cjce-2017-0311.R1 \\
\hline Manuscript Type: & Article \\
\hline Author: & 27-Feb-2018 \\
\hline $\begin{array}{r}\text { Complete List of Authors: } \\
\text { Keyword: }\end{array}$ & $\begin{array}{l}\text { Karimpour, Shooka; York University, Department of Civil } \\
\text { Chu, Vincent H.; Dept. of Civil Engineering and Applied } \\
\text { Mechanic, }\end{array}$ \\
\hline \hline $\begin{array}{r}\text { Is the invited manuscript } \\
\text { for consideration in a } \\
\text { Special Issue? : }\end{array}$ & Not applicable (regular submission) \\
\hline
\end{tabular}




\title{
THE ROLE OF WAVES ON MIXING IN SHALLOW WATERS
}

\author{
Shooka Karimpour and Vincent H. Chu
}

Shooka Karimpour ${ }^{1}$ and Vincent H. Chu. Department of Civil Engineering and Applied Mechanics, McGill University, Montreal, QC, Canada, H3A 0C3

${ }^{1}$ Corresponding author (e-mail: Shooka.Karimpour@mcgill.ca, Phone: +1-514-398-1609). 


\begin{abstract}
The role of waves on mixing is examined in a transverse shear flow in shallow waters for sub-critical, trans-critical, and super-critical flow over a range of convective Froude numbers. At low convective Froude numbers, the rollup of the vortex sheet to form an eddy defines the mixing. The mixing at higher convective Froude numbers, on the other hand, is affected by the shock waves and the radiation of the wave energy from an elongated vorticity element. Significant structural changes of the shear flow occur as the shock waves become discernible in the trans-critical and super-critical range of the convective Froude number. The shear layer growth is restrained while the momentum-thickness to vorticity-thickness ratio increases by a factor greater than 3 . The fractional growth rate of the mixing layer in shallow waters and its dependence on the convective Froude number follows analogously the observed Mach-number dependence in gas dynamics.
\end{abstract}

Key words: Mixing, Transition to Turbulence, Gravity Waves, Shallow Shear Flow, Numerical Simulation.

\title{
1. Introduction
}

Breaking waves of appreciable amplitude are observed in high-speed shear flow in shallow waters, such as in a mountain stream on a steep slope, or a flood current interacting with the obstacles in its path. In the presence of such waves, the mixing of mass and momentum across the shear flow is no longer describable by the conventional theories of turbulence that were developed on the basis of experimental data in shear flow of small Froude numbers. Model simulations of the shear flows in shallow waters are often conducted using the rigid-lid approximation ignoring the ubiquitous presence of the waves. The role of the waves, however, has been examined for the analogous problem in gas dynamics. Studies of the dilation (volume expansion and contraction) effect in gas have revealed the mixing process to depend on the Mach number. Evidence of the dilation effect on turbulence was considered by Bradshaw (1977). The intriguing effect of shock waves on turbulence was examined in a model of the "eddy shocklet" by Oh (1974). Experimental studies by Papamoschou \& Roshko (1988), Samimy \& Elliott (1990), and Rossmann et al. (2002) correlated the spreading rate in the turbulent mixing layer with the value of the convective Mach number. Lee et al. (1991), Vreman et al. (1996), Papamoschou \& Lele (1993), Luo \& Sandham (1994), and Pantano \& Sarkar (2002) among others have conducted numerical simulations to study the Mach-number effect on mixing process. 
Karimpour \& Chu

${ }_{46} U=\frac{1}{2}\left(U_{1}+U_{2}\right)+\frac{1}{2}\left(U_{1}-U_{2}\right) \tanh \frac{2 y}{\delta_{\omega o}}$

47

${ }_{50} \delta_{\omega o}=\frac{U_{1}-U_{2}}{\hat{U}_{y o}}$,

${ }_{52} \quad \hat{U}_{y o}=\left[\frac{d U}{d y}\right]_{y=0, t=0}$

53 The dimensionless parameters for the wave effect are the wave number of the disturbance

${ }_{54} k_{x o}^{*}=\frac{2 \pi \delta_{\omega o}}{\lambda_{x}}$, 

and the convective Froude number

${ }_{56} \quad \mathrm{Fr}_{\mathrm{c}}=\frac{U_{1}-U_{2}}{c_{1}+c_{2}}$

${ }_{61} \mathrm{Ma}_{\mathrm{c}}=\frac{U_{1}-U_{2}}{c_{1}+c_{2}}$

${ }_{66} \frac{\partial h}{\partial t}+\frac{\partial u h}{\partial x}+\frac{\partial h v}{\partial y}=0$

${ }_{68}^{67} \frac{\partial u}{\partial t}+u \frac{\partial u}{\partial x}+v \frac{\partial u}{\partial y}=-g \frac{\partial h}{\partial x}$

69

${ }_{70} \frac{\partial v}{\partial t}+u \frac{\partial v}{\partial x}+v \frac{\partial v}{\partial y}=-g \frac{\partial h}{\partial y}$ of the shallow-water equations

The water depth, $h$, and the velocity components, $u$ and $v$, were determined from numerical solution

where $g$ is the gravity. These depth-averaged equations for the flow in shallow waters are identical in form to the two-dimensional gas-dynamic equations if the specific heat ratio of the gas were $\gamma=2$ (Liggett 1994). The computation was carried out using a fifth-order WENO scheme for the spatial interpolation on a staggered grid and a fourth-order Runge-Kutta method for the time integration. The accuracy of the WENO interpolation and time integration method was determined to be superior over the other numerical schemes in a comparison study of numerical methods by Karimpour \& Chu (2015).

Figure 1 shows the computational domain. It is defined by the periodic boundary condition over one wave length in the longitudinal direction from $x=0$ to $x=\lambda_{x}$, and the radiation boundary condition 
79

${ }_{80} v^{++}=c_{1} \frac{h^{++}-H}{H}$ at $y=y^{++}, \quad v^{--}=c_{2} \frac{h^{--}-H}{H} \quad$ at $\quad y=y^{--}$,

81

$88 \frac{h^{\prime}}{\delta_{\omega o}}=10^{-10} \sin \left\{\frac{2 \pi x}{\lambda_{x}}\right\}$ in $-\lambda_{x} / 64<y<+\lambda_{x} / 64$, and $h^{\prime}=0$ elsewhere

${ }_{97} \bar{U}=\frac{1}{\lambda_{x}} \int_{0}^{\lambda_{x}} u d x$ and $\bar{H}=\frac{1}{\lambda_{x}} \int_{0}^{\lambda_{x}} h d x$

99 increase in the dimension of the domain did not produce detectable difference in the simulation results. central region as follows:

The width of the strip is confined to $\lambda_{x} / 64$ on each side, which is a integer multiple of the grid size in previous study of the linear instability by Karimpour \& Chu (2016). The amplitude of the disturbance eventually becomes large enough to modify the mean flow. Gravity waves of significant amplitude $u, v$, and $h$, include both mean and fluctuating elements and are decomposed as follows:

$h^{\prime}=h-\bar{H}, u^{\prime}=u-\bar{U}$ and $v^{\prime}=v$

The typical vorticity profiles, mean-flow velocity profiles and depth-fluctuation profiles are shown in

in which $h^{++}$and $h^{--}$are the depth at the edge of radiating boundaries. These radiation boundary conditions are imposed so that the waves can escape from the computational domain without the reflection at the domain's boundaries $y^{--}=-3 \lambda_{x}$ and $y^{++}=3 \lambda_{x}$. The domain from $y=-3 \lambda_{x}$ to $y=3 \lambda_{x}$ was sufficiently large compared with the vorticity thickness of the mixing layer. Further The initial disturbances were the small fluctuations in depth introduced along a narrow strip in the all grid resolutions chosen for the grid refinement study. The initial growth of the small disturbance, within a specific range of wave numbers, is exponential in an unstable shear flow as shown in the would be produced if the convective Froude number is large. The mean velocity $\bar{U}$ and the mean depth $\bar{H}$ are nonlinearly modified by the finite-amplitude disturbance. The velocity and depth components, Figures 1 . The first row in the figure shows $\left(a_{1}\right)$ the vorticity contours, $\left(b_{1}\right)$ the mean velocity profile, and $\left(c_{1}\right)$ the depth-fluctuation contours for the super-critical flow with a convective Froude number $\mathrm{Fr}_{\mathrm{c}}$ $=1.4$ and a wave number $k_{x o}^{*}=2 \pi \delta_{w o} / \lambda_{x}=0.35$. The profiles in the second row are $\left(\mathrm{a}_{2}\right)$ the vorticity 
${ }_{119} \overline{\overline{K^{\prime}}}=\frac{1}{\delta_{\omega o} \lambda_{x}} \int_{y^{--}}^{y^{++}} \int_{0}^{\lambda_{x}} \frac{1}{2}\left(u^{\prime 2}+v^{\prime 2}\right) d x d y$

${ }_{121}{\overline{\bar{K}^{\prime}}}^{*}=\frac{\overline{\overline{K^{\prime}}}}{\left(U_{1}-U_{2}\right)^{2}}$ the nonlinear instability on the waves' effect.

\section{Kinetic Energy of the Disturbance}

with the dimensionless time

$t^{*}=t \hat{U}_{y o}$

contours, $\left(b_{2}\right)$ the mean velocity profile, and $\left(c_{2}\right)$ the depth-fluctuation contours for the sub-critical flow with a convective Froude number $\mathrm{Fr}_{\mathrm{c}}=0.1$ and a wave number $k_{x o}^{*}=2 \pi \delta_{w o} / \lambda_{x}=0.89$. The nonlinear development in the super-critical shear flow with the $\mathrm{Fr}_{\mathrm{c}}=1.4$ is associated with a thin and elongated vorticity element and the depth fluctuations $h^{\prime}$ are beyond $25 \%$ of the mean depth $\bar{H}$; see the depth contours in Figure 1 $\left(\mathrm{c}_{1}\right)$. The elongated vorticity element and its waves were examined as an entity referred to as the "eddy-shocklet" in gas dynamics by Oh (1974). The sub-critical mixing with the $\mathrm{Fr}_{\mathrm{c}}=0.1$, on the other hand, is defined by the rollup of vorticity to form the "eddy". The depth fluctuation $h^{\prime}$ in this sub-critical flow is small less than $1 \%$ of its mean depth; see the depth contours in Figure $1\left(c_{2}\right)$. In the subsequent sections, quantitative data are to be presented for the dependence of

A number of flow parameters from the simulation are evaluated for their variations in time during the transition, and the dependence of the variations on the convective Froude number. One parameter of significance is the kinetic energy $\overline{\overline{K^{\prime}}}$ of the disturbance obtained by averaging over the computational domain from $x=0$ to $x=\lambda_{x}$ and then from $y=y^{--}$to $y^{++}$as follows:

The time evolutions of the dimensionless fluctuation kinetic energy

are shown in Figure 2 for three representative shear flows: (a) the sub-critical flow with a convective Froude number $\mathrm{Fr}_{\mathrm{c}}=0.1$ and initial wave number $k_{x o}^{*}=0.89$; (b) the trans-critical flow with $\mathrm{Fr}_{\mathrm{c}}=$ 0.8 and $k_{x o}^{*}=0.51$; and (c) the super-critical flow with $\operatorname{Fr}_{\mathrm{c}}=1.4$ and $k_{x o}^{*}=0.35$. Two instants in the transitional development of the kinetic energy $\overline{\overline{K^{\prime}}}$ are identified. The most rapid increase in the 
${ }_{137} \frac{\sqrt{\left[\overline{\overline{K^{\prime}}}\right]_{R}}}{\left(U_{1}-U_{2}\right)}=0.17,0.20$, and 0.17

${ }_{149} \quad \delta_{\omega}=\frac{U_{1}-U_{2}}{\hat{U}_{y}}$ velocity profile

for the sub-critical flow $\mathrm{Fr}_{\mathrm{c}}=0.1$, the trans-critical flow $\mathrm{Fr}_{\mathrm{c}}=0.8$ and the super-critical flow $\mathrm{Fr}_{\mathrm{c}}=1.4$, respectively. The corresponding root-mean-square (rms) values of the velocity fluctuations are

The level of the velocity fluctuation $\sqrt{\left[\overline{\overline{K^{\prime}}}\right]_{R}} /\left(U_{1}-U_{2}\right)=0.17$ obtained for the sub-critical flow is comparable in magnitude to the laboratory data available for the sub-sonic incompressible flow. The maximum of the root-mean-square $\sqrt{\overline{u^{\prime 2}}} /\left(U_{1}-U_{2}\right)$ and $\sqrt{\overline{v^{\prime 2}}} /\left(U_{1}-U_{2}\right)$ obtained from laboratory measurement in an incompressible mixing layer by Wygnanski \& Fiedler (1970) were 0.176 and 0.138 , respectively. The corresponding root-mean-square values of the velocity fluctuations in the trans-critical and super-critical flow are yet to be determined from laboratory measurements.

\section{Vorticity Thickness and Momentum Thickness}

The mean velocity profile is modified by the mixing during the nonlinear transition. The length scales of the mean flow that increase with the mixing are the vorticity thickness $\delta_{\omega}$ and the momentum thickness $\delta_{\theta}$. The vorticity thickness is defined by the velocity gradient $\hat{U}_{y}$ at the inflection point of the mean- 
${ }_{151} \delta_{\theta}=\int_{y^{--}}^{y^{++}} \frac{\bar{U}-U_{2}}{U_{1}-U_{2}}\left(1-\frac{\bar{U}-U_{2}}{U_{1}-U_{2}}\right) d y$

${ }_{173} r=\frac{\Delta_{k}}{\Delta_{k+1}}=2$ be given in later sections. grid refinement,

The vorticity thickness $\delta_{\omega}$ characterizes the vorticity at the inflection point. The momentum thickness $\delta_{\theta}$ is a measure of momentum deficit due to both the vorticity and the wave formation. The vorticity exists only in the region near the inflection point, while the waves are originated from the mixing layer but observed everywhere in the computational domain. The close relation between the disturbance

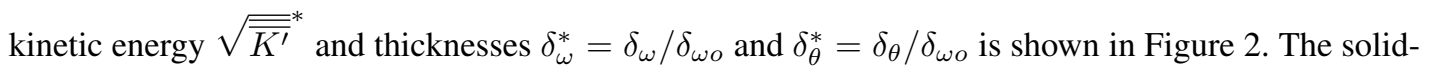
circle symbol defined by maximum growth of $\sqrt{{\overline{\overline{K^{\prime}}}}^{*}}$ at the R-instant of time is shown in the figure to occur at the same time when $\delta_{\omega}^{*}$ and $\delta_{\theta}^{*}$ increase most rapidly. Similarly, the open circles for the peak of ${\sqrt{\overline{\bar{K}^{\prime}}}}^{*}$ at the S-instant of time define also the peaks of $\delta_{\omega}^{*}$ and $\delta_{\theta}^{*}$. The vorticity thickness characterizes the velocity profile near the inflection point. The momentum thickness, on the other hand, is a length scale defined by the entire flow field including the radiation of the waves from the instability. For the imposed initial conditions in section 2 initial ratio of vorticity to momentum thicknesses is given by $\delta_{\omega o}^{*}$ $=4 \delta_{\theta o}^{*}$. Further discussion on the relation between these length scales $\delta_{\omega}$ and $\delta_{\theta}$ and their dependence on the convective Froude number for sub-critical flow, trans-critical flow and super-critical flow will

\section{Computation Accuracy and Grid Refinement}

A note regarding computation accuracy is given in this section before further discussion of the results.

The computations presented in this paper are obtained using a grid of $N=256$ nodes over the wave length $\lambda_{x}$. Table 1 shows the fractional computation errors for the progressively refined grid with $N=$ 64, 128 and 256. The extrapolation for the exact value of $\overline{\bar{K}^{\prime}}{ }_{S}$ for $\Delta x \rightarrow 0$ and $P_{k}$, the order of convergence, are calculated using the extrapolation formulas by Stern et al. (2003). For the present 
${ }_{187} \quad \alpha=\frac{1}{\overline{\overline{K^{\prime}}}} \frac{d \overline{\overline{K^{\prime}}}}{d t}$ growth rate $4 \delta_{\theta}$ as follows:

$\alpha^{*}=\frac{\alpha\left(4 \delta_{\theta}\right)}{\left(U_{1}-U_{2}\right)}$

$k_{x}^{*}=\frac{2 \pi\left(4 \delta_{\theta}\right)}{\lambda_{x}}$

The orders of convergence for the Kinetic Energy of the Disturbance, $\overline{\bar{K}^{\prime}}{ }_{S}$, from the data in Table 1 evaluated by the extrapolation formulas by Stern et al. (2003) are $P_{k}=3.09$ and 3.36 for $\operatorname{Fr}_{\mathrm{c}}=0.8$ and 1.4 , respectively. These orders of convergence are consistent with the gradients of the simulation data plotted in logarithmic scale as shown in Figure 3. The boldface data in the table are the extrapolations to the true values for $\Delta x \rightarrow 0$. The Fractional computational Error in percentage is determined by

$\mathrm{FE}(\%)=\frac{\left|\overline{\overline{K^{\prime}}} S-{\overline{\overline{K^{\prime}}}}_{S_{\Delta x \rightarrow 0} \mid}\right|}{\overline{\overline{K^{\prime}}} S_{\Delta x \rightarrow 0}} \times 100$

Using the $N=256$ nodes, the calculations for the $\overline{\bar{K}^{\prime}}$ are accurate to 4 significant digits. The fractional computation errors are estimated to be $\mathrm{FE}=0.14 \%$ and $0.26 \%$ for $\mathrm{Fr}_{\mathrm{c}}=0.8$ and 1.4 respectively. Full details of the grid-refinement study for the sub-critical flow with $\mathrm{Fr}_{c}<0.8$, were given in a previous paper by Karimpour \& Chu (2015).

\section{Fractional Growth Rate of the Disturbance's Kinetic Energy}

The parameter for both the linear and the nonlinear development of the instability is the fractional

This rate is made dimensionless using the velocity difference $\left(U_{1}-U_{2}\right)$ and the momentum thickness

Figures 4 (a), (b) and (c) are the stability diagrams that show the correlation of this dimensionless growth parameter with the dimensionless wave number

for (a) the sub-critical flow with $\mathrm{Fr}_{c}=0.1$, (b) the trans-critical flow with $\mathrm{Fr}_{c}=0.8$ and (c) the supercritical flow with $\mathrm{Fr}_{c}=1.4$, respectively. The momentum thickness times four, that is the $4 \delta_{\theta}$ used 
in Equation 22 and 23, is equal to the vorticity thickness $\delta_{\omega}$ in the initial linear development when the mean flow is the TANH profile defined by Equation 1. The factor of four is introduced so that the dimensionless parameters $\alpha^{*}$ and $k_{x}^{*}$ are the same as those used in the linear instability analysis by Karimpour \& Chu (2015). In the nonlinear development of the instability, the mean flow is no longer defined by the TANH profile. Both vorticity thickness $\delta_{\omega}$ and momentum thickness $\delta_{\theta}$ increase with time. The relation between $\delta_{\omega}$ and $\delta_{\theta}$ also changes with the nonlinear development of the instability. The $4 \delta_{\theta}$ is used as the length scale to define $\alpha^{*}$ and $k_{x}^{*}$ because the growth in momentum thickness $\delta_{\theta}$ is a representative of the combined effect by both vorticity and waves.

The relation between $\alpha^{*}$ and $k_{x}^{*}$ obtained from the linear instability analysis is the dashed-dotdashed line in Figures 4 (a), (b) and (c). The triangle symbol in the figure marks the most amplifying disturbance of the linear instability. The solid line traces the nonlinear development for the most amplified disturbance. The wave numbers for the most amplifying disturbance for three convective Froude numbers are as follows:

(a) $k_{x o}^{*}=0.89$ for the sub-critical instability with $\mathrm{Fr}_{c}=0.1$

(b) $k_{x o}^{*}=0.51$ for the trans-critical instability with $\mathrm{Fr}_{c}=0.8$

(c) $k_{x o}^{*}=0.35$ for the super-critical instability with $\mathrm{Fr}_{c}=1.4$

Starting the disturbance with a full spectrum of different wave numbers, only the most amplifying disturbances with the wave numbers $k_{x o}^{*}=0.89,0.51$ and 0.35 for $\operatorname{Fr}_{c}=0.1,0.8$ and 1.4, respectively, can fully developed to finite amplitude. Since the wave length is inversely proportional to the wave number according to Equation 23, the wave length of the most amplifying super-critical instability is approximately 2.5 time greater than the wave length of the sub-critical instability. The nonlinear development of $\overline{\overline{K^{\prime}}}$ at the R-instant of time and the S-instant of time are marked on the solid lines by the solid circle and open circle, respectively. The relative wave length $\lambda_{x} /\left(4 \delta_{\theta}\right)$ and $k_{x}^{*}=2 \pi\left(4 \delta_{\theta}\right) / \lambda_{x}$ changes with time as the mean flow is modified by the disturbance of finite amplitude. The wave length $\lambda_{x}$ is constant but the momentum thickness $\left(4 \delta_{\theta}\right)$ changes with time during the nonlinear transition. The most rapid variation of $\overline{\overline{K^{\prime}}}$ and $\left(4 \delta_{\theta}\right)$ occurs at time $t=t_{R}$. The fractional rates of growth of $\overline{\overline{K^{\prime}}}$ at this R-instant of time are

$\alpha_{R}^{*}=0.16,0.071$ and 0.016 
for $\operatorname{Fr}_{c}=0.1,0.8$ and 1.4, respectively. This rate $\alpha_{R}^{*}=0.016$ for the super-critical instability with $\mathrm{Fr}_{c}=1.4$ is an order of magnitude less than the rate $\alpha_{R}^{*}=0.16$ for the sub-critical instability with $\mathrm{Fr}_{c}=0.1$. The radiation of wave energy away from the shear flow and the energy dissipation across the shock waves impede the mixing process in the super-critical flow by a factor of 10 in terms of $\alpha_{R}^{*}$.

\section{Depth and Vorticity Profiles}

The contours for the water depth, $h^{*}=h / \bar{H}$, and the vorticity, $\zeta^{*}=\zeta \delta_{\omega o} /\left(U_{1}-U_{2}\right)$, for sub-critical, trans-critical, and super-critical shear flows with the most amplifying initial disturbance (as specified in section 6) are shown in Figures 5 (a), (b) and (c), respectively. The first two rows in the figure are the depth contours. The depth variation is less than $1 \%$ of the mean water depth for the sub-critical flow. More than 25\% sudden change in water depth across the shock waves is observed in the trans-critical and super-critical shear flows. The vorticity contours also are very different among the sub-critical, trans-critical and super-critical flows as shown in the third and fourth rows in the figure. In the subcritical flow, the exchanges of mass and momentum are associated with the rollup of the vortex sheet in the form of an eddy. In the presence of the shock waves in the trans-critical and super-critical exchanges on the other hand, the vortex sheet tends to stretch out and then break up into elongated elements that slide over each other. The interaction between the shock waves and the vorticity elements is not well understood. Oh (1974) has used the term "eddy-shocklet" to distinguish this not-so-well understood interaction between the vorticity and the shock waves from eddy development alone. The depth-andvelocity discontinuities in the eddy-shocklets are well captured by the numerical simulations conducted using the WENO scheme. While the vorticity is confined within a narrow region in the shear flow, the waves extend far beyond the region. Energy is dissipated locally across the shock waves and at the same time radiated away from the shear flow. The dissipation and the radiation of the wave energy are the processes that take away the available energy for mixing, therefore slowing down the exchanges of mass and momentum across the trans-critical and super-critical shear flows.

\section{Fractional Spreading Rates}

The vorticity thickness $\delta_{\omega}$ and the momentum thickness $\delta_{\theta}$ are length scales often measured in laboratory experiments to characterize the exchanges of mass and momentum across shear flows. The 
${ }_{252} \beta_{\omega}=\frac{1}{\delta_{\omega}} \frac{d \delta_{\omega}}{d t}$ and $\beta_{\theta}=\frac{1}{\delta_{\theta}} \frac{d \delta_{\theta}}{d t}$

${ }_{254} \beta_{\omega}^{*}=\frac{\beta_{\omega} \delta_{\omega o}}{\left(U_{1}-U_{2}\right)}$ and $\beta_{\theta}^{*}=\frac{\beta_{\theta} \delta_{\omega o}}{\left(U_{1}-U_{2}\right)}$ sub-critical values

$\frac{\beta_{\omega R}}{\beta_{\omega R_{0.1}}}$ and $\frac{\beta_{\theta R}}{\beta_{\theta R_{0.1}}}$ well with the Langley experimental curve.

fractional rates of increase in these length scales due to the exchanges are the $\beta$-coefficients:

The dimensionless expressions of these spreading coefficients are

Figure 6(a) shows the dependence of these spreading coefficients $\beta_{\omega R}^{*}$ and $\beta_{\theta R}^{*}$ for the most amplified disturbances at time $t=t_{R}$ on the convective Froude number. The dissipation and radiation of wave energy from the instability has led to significant reduction in value of these coefficients in the transcritical and super-critical flows. Figure 6(b) shows values of the spreading coefficients relative to their

The normalization is based on the values of $\beta_{\omega R_{0.1}}$ and the $\beta_{\theta R_{0.1}}$ obtained for $\operatorname{Fr}_{\mathrm{c}}=0.1$. The correlation of these normalized coefficients with the convective Froude number in this figure compares well with the Langley experimental curve that was obtained from an analogous correlation of compressible air flow data with the convective Mach number. The dashed line in this figure represents results from the Langley experiment. This curve is extracted and reproduced by the authors from Pantano \& Sarkar (2002). The reduction of the spreading-rate coefficients with the convective Froude number is associated with the slowing down of the mixing process and the increase in the wave activity. There is no experimental study of the wave effect on shallow waters. However, the numerical and laboratory investigations of compressible shear flow in air by Samimy \& Elliott (1990), Pantano \& Sarkar (2002), and Gatski \& Bonnet (2013) have produced data for the fractional spreading rate that also compare 
${ }_{275} \mathrm{SF}=\frac{4 \delta_{\theta}}{\delta_{\omega}}$ factor

\section{Shape Factor}

The parameter that defines the modification to the mean flow by the presence of the waves is the shape

The shape factor is $\mathrm{SF}=1$ for the undisturbed TANH velocity profile. Figure 7 (a) shows the variation of this shape factor with the convective Froude number. The solid symbol denotes the values of the shape factor $\mathrm{SF}_{R}$ at time $t=t_{R}$ while the open symbol is the shape factor $\mathrm{SF}_{S}$ at time $t=t_{S}$. For the sub-critical flow in the range from $\mathrm{Fr}_{\mathrm{c}}=0.1$ to 0.65 , the value of the shape factor is $\mathrm{SF}_{R} \simeq 0.8$. This shows that the mean velocity profile of the sub-critical flow in the presence of the finite amplitude disturbance is not the TANH profile. The value of the shape factor increases significantly from $\mathrm{SF}_{R} \simeq$ 0.8 to 2.5 in the trans-critical flow as the convective Froude numbers increases from $\operatorname{Fr}_{\mathrm{c}} \simeq 0.65$ to 1.0. The significant increase in the shape factor, from its sub-critical value of 0.8 to the super-critical value of 2.7, shows clearly the structural changes of the flow in the presence of the waves. The shear flow is classified by the shape factor (a) as sub-critical for the convective Froude number varying from $\mathrm{Fr}_{\mathrm{c}}$ $=0$ to 0.65 , (b) as trans-critical for $\mathrm{Fr}_{\mathrm{c}}=0.65$ to 1.0 , and (c) as super-critical when the value of $\mathrm{Fr}_{\mathrm{c}}$ exceeds unity. This classification is consistent with the dissipation and the radiation of the energy to be evaluated and explained in the subsequent sections that follow similar dependence on $\mathrm{Fr}_{\mathrm{c}}$ as shown in Figure 7 (b) and (c).

\section{Elongated Structure of the Eddy-Shocklet}

A direct measure of the shape of the eddy and the eddy-shocklet is the width-to-length ratio $4 \delta_{\theta} / \lambda_{x}$, which at time $t=t_{R}$ is

$\left[\frac{4 \delta_{\theta}}{\lambda_{x}}\right]_{t=t_{R}}=0.199,0.151,0.075$

for $\mathrm{Fr}_{\mathrm{c}}=0.1,0.8$ and 1.4, respectively. These values are obtained from the simulation and defined by the solid circles shown in Figure 2 (a), (b), and (c). The corresponding width-to-length ratio for the 
$298\left[\frac{\delta_{\omega R}}{\lambda_{x}}\right]_{t=t_{R}}=\left[\frac{4 \delta_{\theta R}}{\lambda_{x}} \frac{1}{S F}\right]_{t=t_{R}}=0.249,0.068$ and 0.028 ,

$320 \quad e=\frac{1}{2} \rho\left(u^{2}+v^{2}\right) h+\frac{1}{2} \rho g h^{2}$

$\left[\frac{4 \delta_{\theta}}{\lambda_{x}}\right]_{t=t_{S}}=0.255,0.201$ and 0.118

respectively. For the sub-critical flow with $\mathrm{Fr}_{\mathrm{c}}=0.1$ and $\lambda_{x} / \delta_{\omega R} \simeq 0.249^{-1} \simeq 4$, the eddy is approximately 4 times greater in length compared with its width. This shape of the eddy is in agreement with the flow visualization of the coherent structure in the mixing layer by Brown and Roshko (1974). The length-to-width ratio of the super-critical mixing layer, on the other hand, is an order of magnitude greater. For the super-critical flow with $\mathrm{Fr}_{\mathrm{c}}=1.4$, the ratio $\left[\lambda_{x} / \delta_{\omega R}\right]=0.028^{-1} \simeq 36$. The highly elongated vorticity elements in the eddy-shocklet for the trans-critical and the super-critical flow is already delineated in Figures 1 and 5. The width-to-length ratio at time $t=t_{S}$

for $\mathrm{Fr}_{\mathrm{c}}=0.1,0.8$ and 1.4, respectively. These values are obtained from the simulation defined by the open circles shown in Figure 2 (a), (b), and (c). They are associated with the maximum of $\delta_{\omega}$ when $\alpha_{S}^{*}$ vanishes to zero. Further increase in the width-to-length ratio beyond these values is not possible without the merging of the eddy and the eddy-shocklet with their neighbors.

\section{Wave Energy Consideration}

The shock waves with sudden changes in depth and velocity are responsible for the local energy dissipation. The waves, in general, are responsible for the radiation of the energy away from the region where the disturbance's energy is originally produced. As a consequence of dissipation and radiation, the energy level available for the mass and momentum exchanges across the shear flow and growth across the mixing layer is reduced. The role of the waves on shear instability and mixing layer development in this study is investigated by considering the energy balance from the numerical data. We begin the consideration by first deriving the shallow-water energy equation. The kinetic energy plus potential energy per unit area of the shallow water, with density of $\rho$, is 
Karimpour \& Chu

321 The flux of the energy is

322 $\overrightarrow{\mathcal{F}}=\frac{1}{2} \rho(\vec{U} h)\left(u^{2}+v^{2}\right)+\rho(\vec{U} h) g h$

323

324

${ }_{325} \frac{\partial e}{\partial t}+\nabla \cdot \overrightarrow{\mathcal{F}}=0$

326

327

${ }^{329} \int_{A} \frac{\partial e}{\partial t} d A+\int_{A} \nabla \cdot \overrightarrow{\mathcal{F}} d A=0$

330

${ }_{331} \frac{\partial}{\partial t} \int_{A} e d A+\oint_{S} \overrightarrow{\mathcal{F}} \cdot \hat{n} d S=-\int_{A} \epsilon d A$

$\underbrace{\frac{\partial}{\partial t}\left(\int_{y^{-}}^{y^{+}} \int_{0}^{\lambda_{x}} e d x d y\right)}_{[\mathrm{I}]}+\overbrace{\left[\int_{0}^{\lambda_{x}} \mathcal{F} d x\right]_{\text {at } y=y^{+}}-\left[\int_{0}^{\lambda_{x}} \mathcal{F} d x\right]_{\text {at } y=y^{-}}}^{-[\mathrm{II}]}=-\underbrace{\int_{A} \epsilon d A}_{[\mathrm{III}]}$

This energy equation has been derived by Majda (2003) among others who studied the shear flow in shallow waters. To study the role of the waves, the energy equation is integrated over an interrogation zone of area $A$ and perimeter $S$ :

Interchanging the order of differentiation and integration,

where $\epsilon$ is the energy dissipation per unit surface area of the flow in shallow water, and $\hat{n}$ is the unit vector in the direction perpendicular to the perimeter of the interrogation zone. The energy dissipation term is produced by interchanging the order of differentiation and integration across the depth and velocity discontinuities. The interrogation zone is an area bounded by lines located at $y=y^{-}$and $y=y^{+}$from $x=0$ to $x=\lambda_{x}$, equally spaced from the mixing layer. The perimeter along the periodic boundary makes no net contribution to the fluxes. The unit vector $\hat{n}$ is in the negative $y$-direction at the perimeter where $y=y^{-}$, and in the positive $y$-direction at $y=y^{+}$. Therefore, $\vec{U} \cdot \hat{n}=-v$ at $y=y^{-}$ and $\vec{U} \cdot \hat{n}=+v$ at $y=y^{+}$and with these the integral energy equation becomes 
in which

$\mathcal{F}=\frac{1}{2} \rho v h\left(u^{2}+v^{2}\right)+\rho v g h^{2}$

is the flux of energy through the perimeter of the interrogation zone. Terms [I], [II] and [III] are calculated directly from the numerical simulation. Term [II] is the sum of all energy fluxes in and out of the interrogation zone. The difference between term [I] and term [II] gives the energy dissipation term [III]. In sub-critical flow without the waves, the dissipation term [III] $=[\mathrm{I}]-[\mathrm{II}]$ is almost zero according to the evaluation of the term using the simulation data.

\section{Radiation Energy Flux}

The energy flux that is, term [II] in Equation 37, is calculated using two interrogation zones to observe it propagation with the waves of celerity $c \simeq \sqrt{g H}$. Figure 8 shows the dimensionless expression of the flux

$[\mathrm{II}]^{*}=\frac{[\mathrm{II}]}{\lambda_{x} \rho H c\left(U_{1}-U_{2}\right)^{2}}$

plotted versus time $t^{*}$ for the three typical cases: (a) sub-critical instability with $\operatorname{Fr}_{c}=0.1$ and $k_{x o}^{*}=$ 0.89 , (b) trans-critical instability with $\mathrm{Fr}_{c}=0.8$ and $k_{x o}^{*}=0.51$, and (c) super-critical instability with $\mathrm{Fr}_{c}=1.4$ and $k_{x o}^{*}=0.35$. This energy flux depends on the location of the edges of the interrogation zone at $y=y^{+}$and $y=y^{-}$where the flux is calculated. The edges of one interrogation zone are located at $y^{+}=-y^{-}=4 \delta_{\omega o}$. The edges of the other interrogation zone are located further away at $y^{+}=-y^{-}=12 \delta_{\omega o}$. The red line in the top row in Figure 8 is the flux evaluated at the edges $y=y^{+}=12 \delta_{\omega o}$ of one interrogation zone and the black line is the flux evaluated at the edges at $y=y^{-}=-4 \delta_{\omega o}$ of a smaller interrogation zone. As the wave is propagated with the speed $c$, the arrival of the peak of the flux at the edges further away from the production occurs at a later time. The time-lag difference is $t_{l}=\left(12 \delta_{\omega o}-4 \delta_{\omega o}\right) / \sqrt{g H}$. The first peak of this radiation flux $[\mathrm{II}]_{R}^{*}$ is associated with the initiation of the waves by the instability at time $t=t_{R}$. The black solid circle in Figure 8 marks the magnitude of this peak [II] $]_{R}^{*}$ which occurs at time $t_{R}+4 \delta_{\omega o} / \sqrt{g H}$ at a position $y^{+}=-y^{-}=4 \delta_{\omega o}$. The red solid circle indicates a radiation flux of approximately the same magnitude at a later time $t=t_{R}+12 \delta_{\omega o} / \sqrt{g H}$ further away at a position $y^{+}=12 \delta_{\omega o} . \quad$ The 
energy flux is significant for the trans-critical flow and super-critical flow but is negligibly small for the sub-critical flow with little waves. Figure 7 (c) shows the dependence of the energy flux $[\mathrm{II}]_{R}^{*}$ on the convective Froude number $\mathrm{Fr}_{\mathrm{c}}$. The value of $[\mathrm{II}]_{R}^{*}$ increases almost linearly with the increase of the convective Froude number in the range from $\mathrm{Fr}_{\mathrm{c}}=0$ to 1 . It reaches a relatively more constant value of $[\mathrm{II}]_{R}^{*} \simeq 0.016 \pm 0.004$ for the super-critical flow.

\section{Energy Dissipation}

The energy dissipation term, $[\mathrm{III}]=[\mathrm{I}]-[\mathrm{II}]$ in Equation 37 evaluated from the simulation data, constitutes the second row in Figure 8. The dimensionless expression of the energy dissipation

$[\mathrm{III}]^{*}=\frac{\{[\mathrm{I}]-[\mathrm{II}]\} \delta_{\omega o}}{\left(U_{1}-U_{2}\right)^{2} H c}$

is plotted versus time $t^{*}$ for the (a) sub-critical instability with $\mathrm{Fr}_{c}=0.1$ and $k_{x o}^{*}=0.89$, (b) transcritical instability with $\mathrm{Fr}_{c}=0.8$ and $k_{x o}^{*}=0.51$, and (c) super-critical instability with $\mathrm{Fr}_{c}=1.4$ and $k_{x o}^{*}=0.35$. These energy dissipation rates in time are obtained using two interrogation zones of sizes $y^{+}=-y^{-}=4 \delta_{\omega o}$ and $y^{+}=-y^{-}=12 \delta_{\omega o}$. The energy dissipation for the sub-critical flow of $\operatorname{Fr}_{\mathrm{c}}=$ 0.1 is, as expected, negligibly small. The energy dissipation for the trans-critical flow and super-critical flow is associated with the formation of the shock waves across the depth and velocity discontinuities.

The energy dissipation occurred primarily in the central region between $y=4 \delta_{\omega o}$ and $y=-4 \delta_{\omega o}$. The difference in the dissipation rates obtained in two interrogation zones of very different sizes is quite negligible. We have nevertheless used the larger interrogation zone between $y=12 \delta_{\omega o}$ and $y=-12 \delta_{\omega o}$ to evaluate the energy dissipation rate $[\mathrm{IIII}]_{R}^{*}$ and $[\mathrm{III}]_{S}^{*}$, at the R-instant and S-instant of time, respectively. The energy dissipation rates obtained in this manner are compared with the shape factor SF and the radiation energy flux [II] ${ }^{*}$ in Figure 7 for the classification of the flow. The instability is sub-critical if $\mathrm{Fr}_{c}<0.8$, trans-critical if $0.8<\mathrm{Fr}_{c}<1.0$ and super-critical if $\mathrm{Fr}_{c}>1.0$. The similarity in the dependence of the shape factor SF, the energy flux [II]* and the energy dissipation $[\mathrm{III}]^{*}$ on the convective Froude number is remarkable. It is clear that the structural changes from subcritical flow to trans-critical flow and super-critical flow are consistently related to the production of the waves and the radiation and dissipation of the energy by the waves. 


\section{Trace-back of Radiation Maximum to Most Rapid Growth at the Source}

The association of energy flux with the wave propagation is significant in studying the role of waves on shear instability. Figure 9 (a) traces the radiation flux $[\mathrm{III}]^{*}$ back to the time when the waves are produced at their source near the inflection point $(y \simeq 0)$, where the shear rate is maximum. The black line in the figure is the radiation flux observed at the edge of the interrogation zone $y^{+}=-y^{-}=4 \delta_{\omega o}$. The red line is radiation flux observed some time later and further away, at the edge of the interrogation $y^{+}=-y^{-}=12 \delta_{\omega o}$. The peaks of the radiation flux marked by the solid red circle symbol and the solid black circle symbol do not occur at the same time as shown in Figure 8. Their occurrence, however, is traceable to the maximum growth of the mixing-layer thickness at the source. The trace-back time in Figure 9 (a) is

$t_{\text {traceback }}=t-\frac{4 \delta_{\omega o}}{\sqrt{g H}}$

for the radiation flux observed at $y^{+}=-y^{-}=4 \delta_{\omega o}$, and is

$t_{\text {traceback }}=t-\frac{12 \delta_{\omega o}}{\sqrt{g H}}$

for the flux observed at $y^{+}=-y^{-}=12 \delta_{\omega o}$. With the trace-back, the peaks of radiation flux [II] ${ }^{*}$ as marked by the solid red circle and the solid black circle in Figure 9 (a) now coincide with the R-instant marked by the black circle in Figure 9 (b) when the momentum thickness is growing at its maximum rate. While the peaks of the radiation flux occur at different times depending on the edge location of the interrogation zone, the tracing back in time shows that the peaks of the radiation flux was closely associated with the rapid growth of the mixing-layer thickness. This correlation of the energy flux with the the trace-back time demonstrates the association of the energy flux with the wave propagation. It also explains that the energy available for mixing is reduced due to the wave energy radiating away from the source in trans-critical and super-critical shear flows. The local energy dissipation across the shock wave also reduces the energy available for the exchange of mass and momentum across the shear flow. 


\section{Conclusion}

We examined the role of the waves in the nonlinear transition of the shear instability in shallow waters. In sub-critical flow, the rollup of the vortex sheet to form an eddy defines the nonlinear transition. In trans-critical and super-critical flow, on the other hand, the vorticity is confined and appears as elongated elements. Some of the energy is radiating away and dissipated locally by the breaking of the waves in trans-critical and super-critical flows, which otherwise could have been available for the nonlinear development of the instability in transition. The fractional growth of the shear-flow thickness obtained from the simulation and its rate dependence on the convective Froude number are in close agreement with laboratory observations of analogous mixing in gas dynamics. The simulation is carried out in this paper for a simple shear flow that evolved from the TANH velocity profile. But the numerical method is equally applicable to simulate other related physical problems in high-speed open-channel flow, including rock-and-sediment transport in mountain streams on a steep slope, and the interaction of flood flow with critical infrastructure in its path.

The eddy and the eddy-shocklet in the transition do not have the fine-scale motion that would be found in fully developed turbulent flow. The coherent structure derived from the instability calculations nevertheless reproduced the dominant mixing across the shear flow in agreement with available experimentally observed data. The 'cascade to higher wave numbers' which fills out the high-wave number end of the spectrum of eddy sizes is the 'internal instabilities' and can be assumed to have only a minor role. According to the large-coherent-eddy hypothesis of Brown \& Roshko (1974), the direct numerical simulation should be acceptable for most purposes in hydro-technical and environmental engineering applications. However, while the fine scales due to internal instability may be ignored, the waves should be correctly simulated as they are the dominant component in trans-critical and supercritical shear flow.

\section{References}

Bradshaw , P. 1977 Compressible turbulent shear layers. Annual Review of Fluid Mechanics 9 (1), $33-52$.

Brown, G. L. \& Roshro, A. 1974 On density effects and large structure in turbulent mixing layers. 


\section{J. Fluid Mechanics 64 (4), 775-816}

EKATERINARISI, J. 2005 High-order accurate, low numerical diffusion methods for aerodynamics. Progress in Aerospace Sciences 41 (3-4), 192-300.

Gatski, T. B. \& Bonnet, J. P. 2013 Compressibility, Turbulence and High Speed Flow. Academic Press, 328 pp.

KARIMPOUR, S. \& CHU, V. H. 2015 High-order interpolation schemes for shear instability simulations. International Journal of Numerical Methods for Heat \& Fluid Flow 25 (6), 1340-1360.

KARIMPOUR, S. \& CHU, V. H. 2016 Instability of unbounded transverse mixing layer in shallow waters. Canadian Journal of Civil Engineering 43 (6), 504-510.

LeE, S., LELE, S. K. \& MoIN, P. 1991 Eddy shocklets in decaying compressible turbulence. Physics of Fluids A: Fluid Dynamics (1989-1993), 3 (4), 235-258.

LigGetT, J. A. 1994 Fluid Mechanics. McGraw-Hill Higher Education.

LIN, C. C. 1953 On the stability of the laminar mixing region between two parallel streams in a gas. No. NACA-TN-2887, Washington DC.

Lou, K. H. \& SAndham, N. D. 1994 On the formation of small scales in a compressible mixing layer. Direct and Large-Eddy Simulation I, Springer Netherlands.

MAJDA, A. 1994 Introduction to PDEs and Waves for the Atmosphere and Ocean. Vol. 9. Courant Lecture Notes in Mathematics, American Mathematical Soc., 234 pp.

OH, Y. H. 1974 Analysis of two-dimensional free turbulent mixing. 7th Fluid and Plasma Dynamics Conference. 13 pp.

PANTANO, C. \& SARKAR, S. 2002 Evolution and growth of large-scale structures in high compressibility mixing layers. Journal of Fluid Mechanics, 451, 329 - 371.

Papamoschou, D. \& Lele, S. 1993 Vortex induced disturbance field in a compressible shear layer. Physics of Fluids A: Fluid Dynamics (1989-1993), 5 (6), 1412-1419. 
Karimpour \& Chu

Papamoschou, D. \& RoshKo, A. 1988 The compressible turbulent shear layer: an experimental study. Journal of Fluid Mechanics, 197, 453 - 477.

Rossmann, T., Mungal, M. G. \& Hanson, R. K. 2002 A study of compressibility effects in the high-speed turbulent shear layer using direct simulation. J. Turbulence, 009:1 - 19.

Samimy, M. \& ElliotT, G. S. 1990 Effects of compressibility on the characteristics of free shear layers. AIAA Journal, 28 (3), 439-445.

Sandham, N. D. \& Reynolds, W. C. 1990 Compressible mixing layer-linear theory and direct simulation. AIAA Journal, 28 (4), 618-624.

Stern, F., Wilson, R. V., Coleman, H. W., Paterson, E. G. 2003 Comprehensive approach to verification and validation of CFD simulations - part 1: methodology and procedures. J. Fluids Eng., 123 (4), 793-802.

VReman, A. W., Sandham, N. D. \& LuO, K. H. 1996 Compressible mixing layer growth rate and turbulence characteristics. Journal of Fluid Mechanics, 320, 235-258.

Wygnanski, I. \& FiedLeR 1970 The two-dimensional mixing region. J. Fluid Mechanics 41, $327-$ 361 
Fig. 1. Computational domain defined by the periodic boundary conditions at $x=0$ and $x=\lambda_{x}$ and the radiating boundary conditions at $y=y^{--}$and $y=y^{++}$.

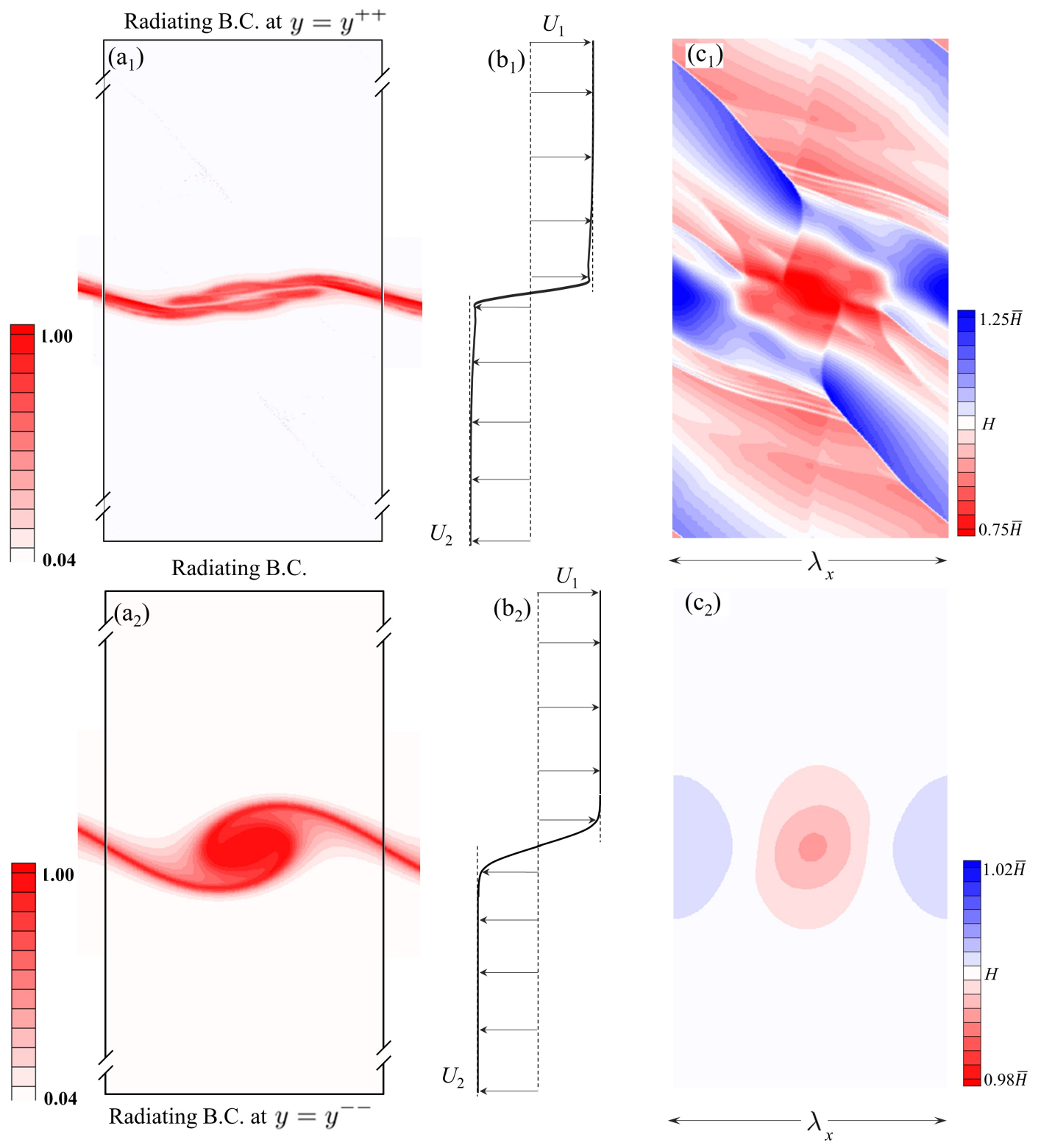


Fig. 2. The evolution of the disturbance's kinetic energy ${\overline{\bar{K}^{\prime}}}^{*}$ (top row), the vorticity thickness $\delta_{\omega}^{*}$ (2nd row) and the momentum thickness $\delta_{\theta}^{*}$ (3rd row) with time $t^{*}$ for (a) the sub-critical flow with $\operatorname{Fr}_{\mathrm{c}}=0.1$ and $k_{x o}^{*}=0.89$, (b) the trans-critical flow with $\operatorname{Fr}_{\mathrm{c}}=0.8$ and $k_{x o}^{*}=0.51$, and (c) the super-critical flow with $\operatorname{Fr}_{\mathrm{c}}=1.4$ and $k_{x o}^{*}=$ 0.35 .

(a) $\mathrm{Fr}_{\mathrm{c}}=0.1, k_{x o}^{*}=0.89$
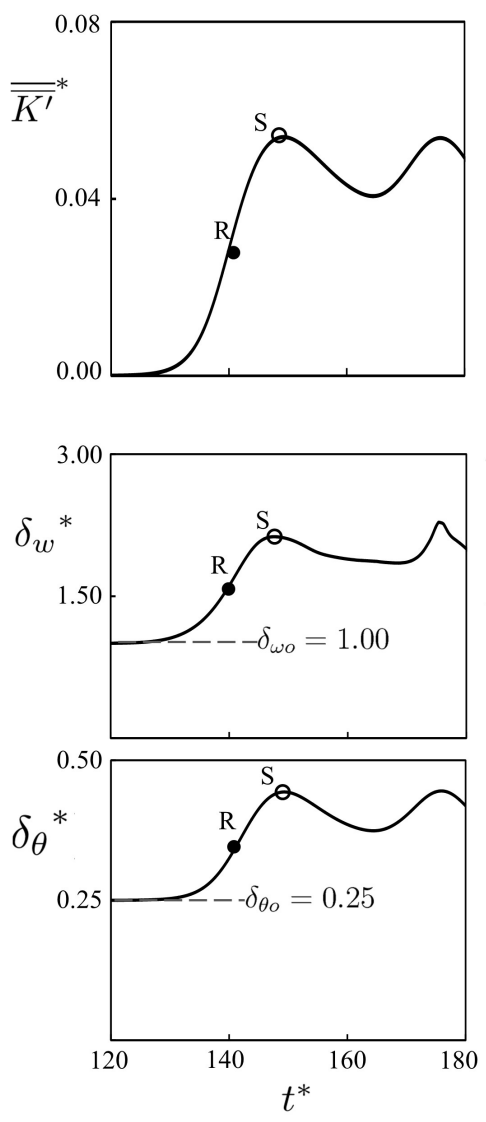

(b) $\mathrm{Fr}_{\mathrm{c}}=0.8, k_{x o}^{*}=0.55$

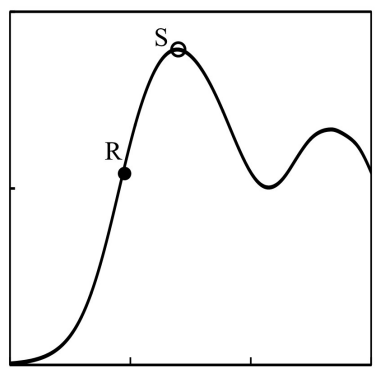

(c) $\mathrm{Fr}_{\mathrm{c}}=1.4, k_{x o}^{*}=0.35$
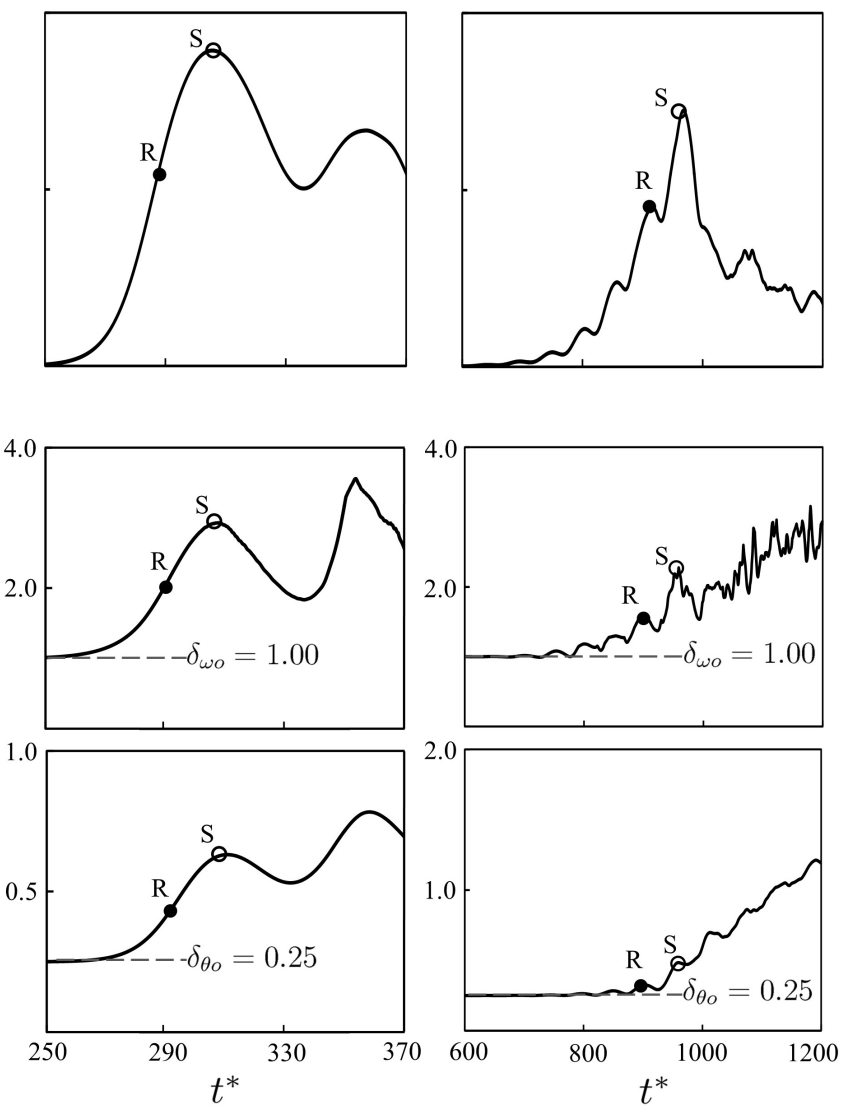

Published by NRC Research Press 
Table 1. Fractional computational error of ${\overline{\overline{K^{\prime}}}}_{S}^{*}$ for the progressively refined grid of $N=64,128$ and 256 for $\mathrm{Fr}_{\mathrm{c}}=0.8$ and 1.4. The bold-faced values are the extrapolations for the exact solutions.

\begin{tabular}{c|cccccc}
$\mathrm{Fr}_{\mathrm{c}}$ & $k_{x}$ & $N$ & $\delta_{\omega o} / \Delta x$ & ${\overline{\overline{K^{\prime}}}}_{S}^{*}$ & $\mathrm{FE}_{s}(\%)$ & Order $\left(P_{k}\right)$ \\
\hline 0.80 & 0.51 & 64 & 5.15 & 0.064084 & 10.22 & - \\
0.80 & 0.51 & 128 & 10.31 & 0.070526 & 1.20 & 3.09 \\
0.80 & 0.51 & 256 & 20.62 & 0.071281 & 0.14 & - \\
0.80 & & & & $\mathbf{0 . 0 7 1 3 8 1}$ & & \\
\hline 1.40 & 0.35 & 64 & 3.57 & 0.041852 & 27.99 & - \\
1.40 & 0.35 & 128 & 7.14 & 0.056536 & 2.72 & 3.36 \\
1.40 & 0.35 & 256 & 14.28 & 0.057966 & 0.26 & - \\
1.40 & & & & $\mathbf{0 . 0 5 8 1 1 9}$ & &
\end{tabular}

Fig. 3. Fractional computational error in percent of ${\overline{\bar{K}^{\prime}}}_{S}^{*}$ for the progressively refined grid of $N=64,128$ and 256 for (a) $\mathrm{Fr}_{\mathrm{c}}=0.8$ and (b) $\mathrm{Fr}_{\mathrm{c}}=1.4$
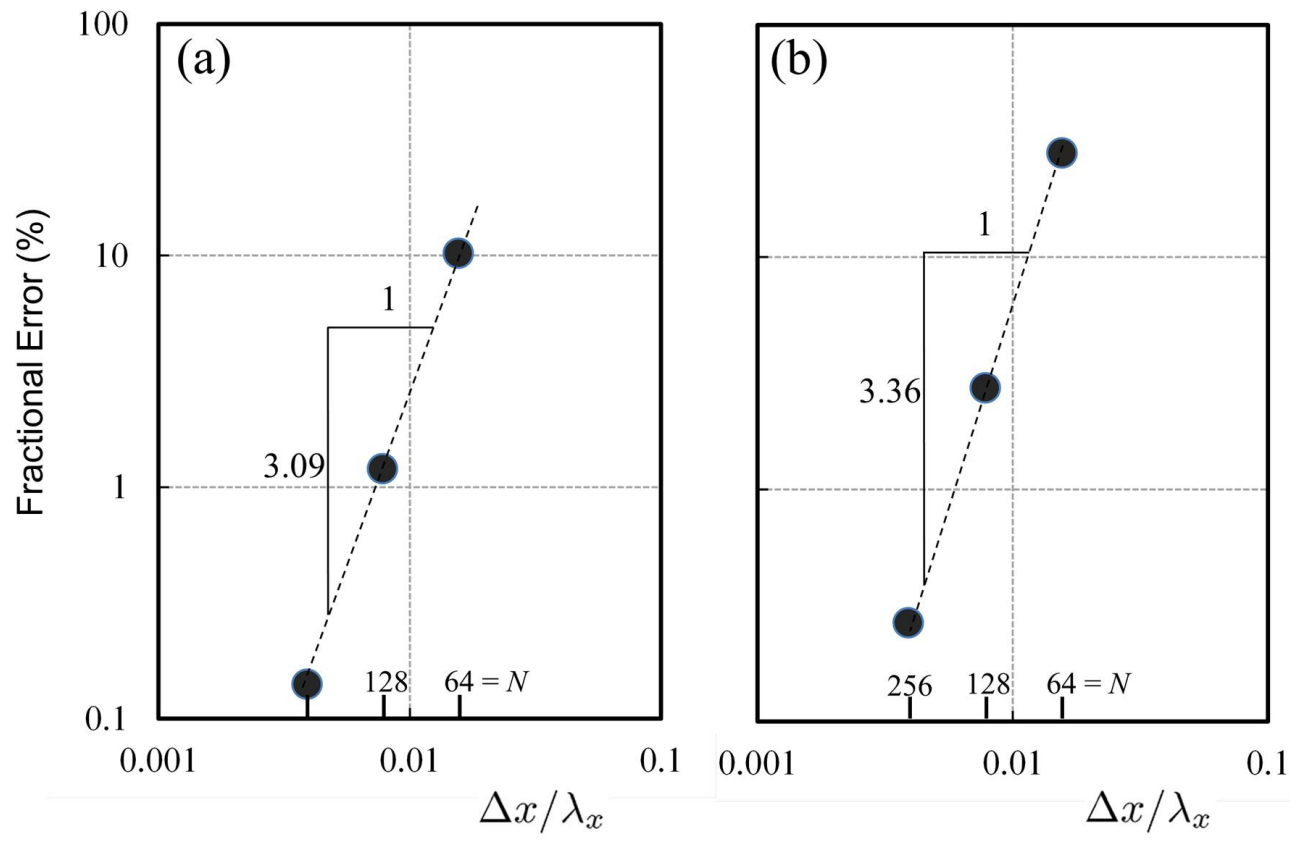
Fig. 4. Variation of the fractional growth rate $\alpha^{*}=\alpha\left(4 \delta_{\theta}\right) /\left(U_{1}-U_{2}\right)$ with the wave number $k_{x}^{*}=2 \pi\left(4 \delta_{\theta}\right) / \lambda_{x}$ for (a) the sub-critical flow with $\mathrm{Fr}_{\mathrm{c}}=0.1$ and $k_{x o}=2 \pi \delta_{\omega o} / \lambda_{x}=0.89$, (b) the trans-critical flow with $\mathrm{Fr}_{\mathrm{c}}=$ 0.8 and $k_{x o}^{*}=2 \pi \delta_{\omega o} / \lambda_{x}=0.51$, and (c) the super-critical flow $\operatorname{Fr}_{\mathrm{c}}=1.4$ and $k_{x o}^{*}=2 \pi \delta_{\omega o} / \lambda_{x}=0.35$.. The dot-dashed line delineates the rate determined by Karimpour \& Chu (2016) for the linear instability. The nonlinear transition follows the solid line that starts at the I-instant for the most amplifying disturbance. It passes through the most rapid increase of ${\overline{\bar{K}^{\prime}}}^{*}$ at the R-instant of time and then settles on to the peak value of ${\overline{\bar{K}^{\prime}}}^{*}$ at the S-instant of time.
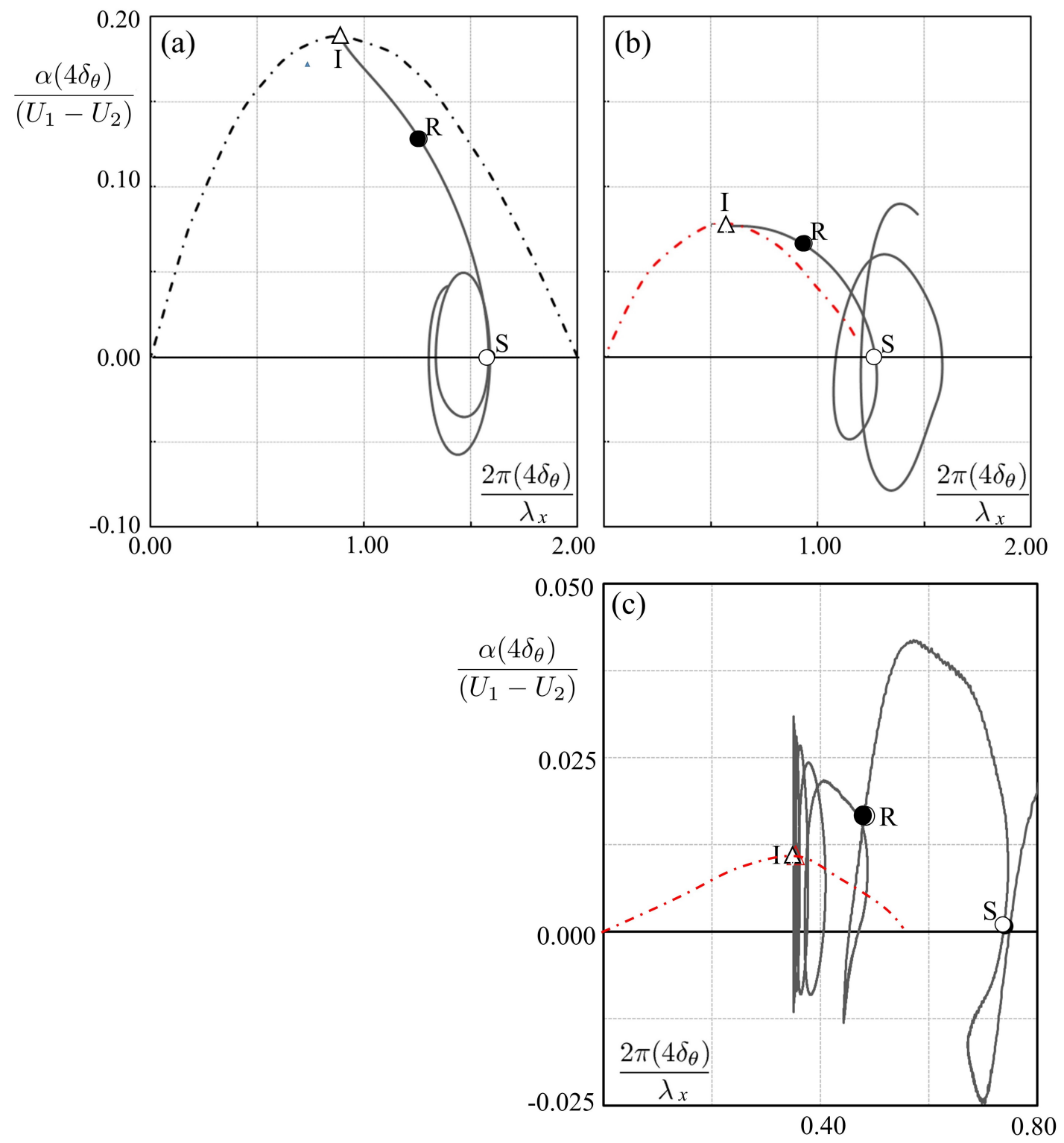

Published by NRC Research Press 
Fig. 5. Dimensionless water-depth contours $h^{*}$ at time $t=t_{R}$ (top row) and at time $t=t_{S}$ ( $2^{\text {nd }}$ row), and dimensionless vorticity contours $\zeta^{*}$ at time $t=t_{R}$ ( $3^{\text {rd }}$ row) and at time $t=t_{S}$ ( $4^{\text {th }}$ row) for (a) the subcritical flow with $\mathrm{Fr}_{\mathrm{c}}=0.1$ and $k_{x o}^{*}=0.89$, (b) the trans-critical flow with $\mathrm{Fr}_{\mathrm{c}}=0.8$ and $k_{x o}^{*}=0.51$, and (c) the super-critical flow with $\operatorname{Fr}_{\mathrm{c}}=1.4$ and $k_{x o}^{*}=0.35$.

(a) $\mathrm{Fr}_{\mathrm{c}}=0.1, k_{x o}^{*}=0.89$
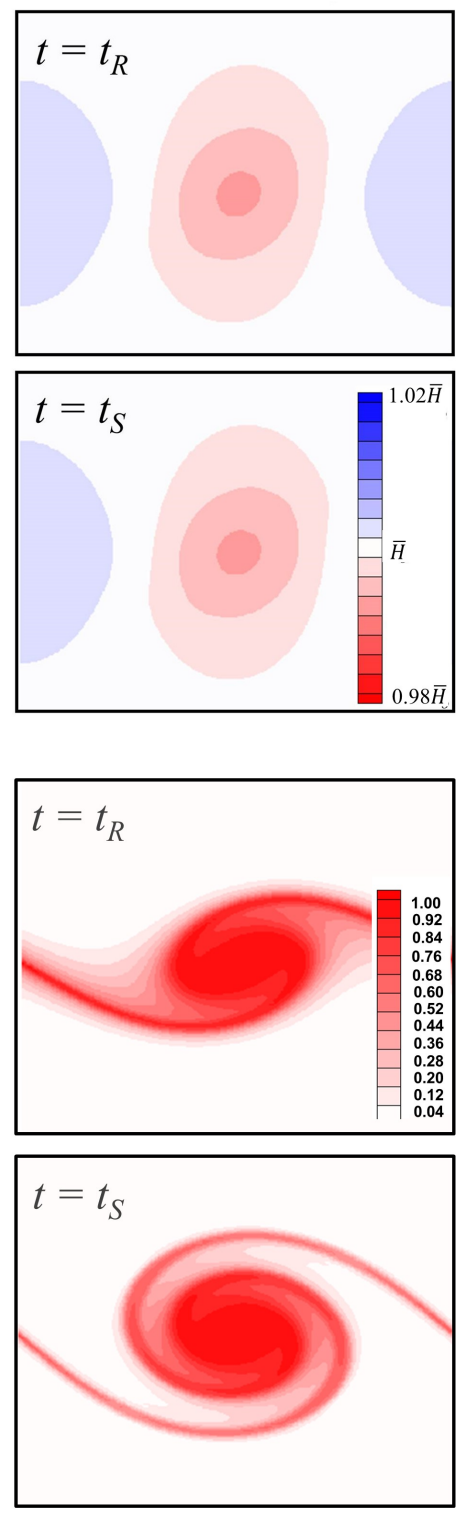

(b) $\mathrm{Fr}_{\mathrm{c}}=0.8, k_{x o}^{*}=0.55$
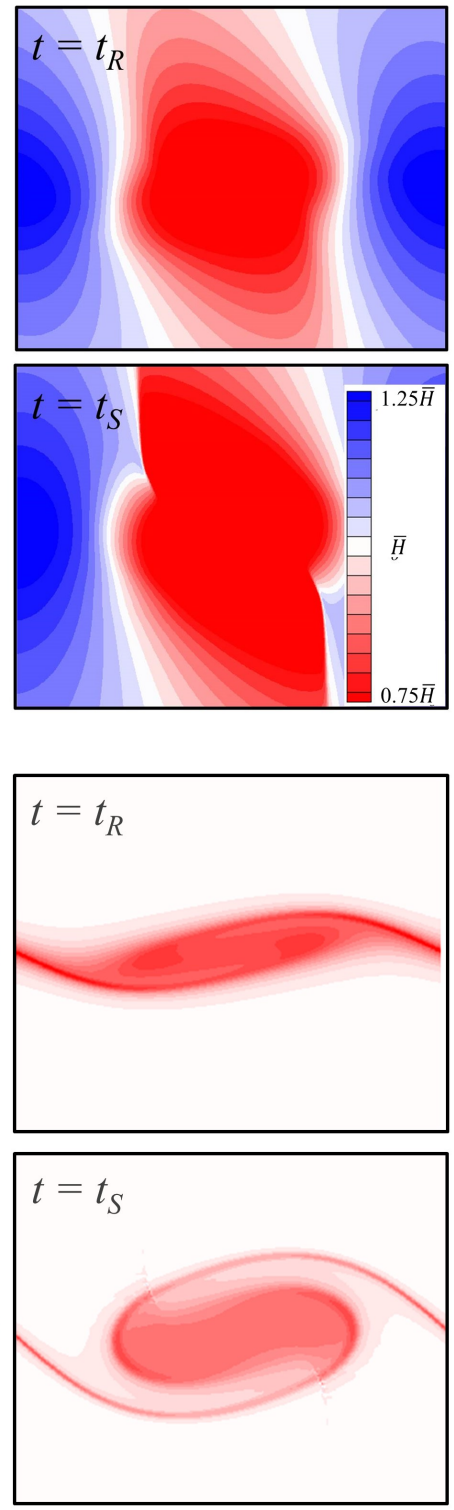

(c) $\mathrm{Fr}_{\mathrm{c}}=1.4, k_{x o}^{*}=0.35$
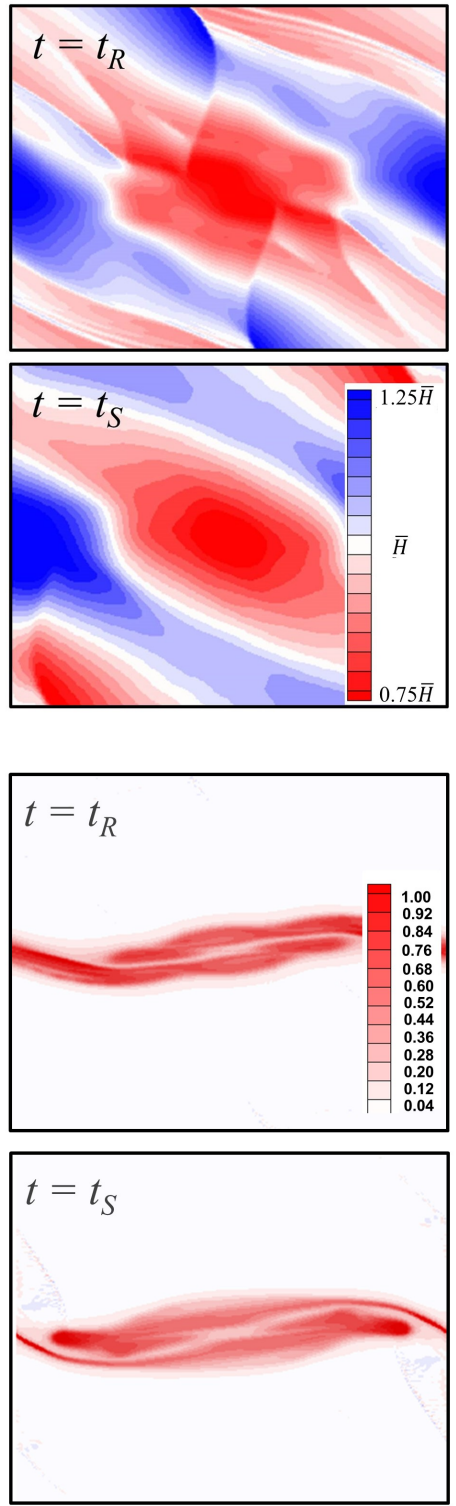
Fig. 6. (a) The dependence of the fractional spreading rate on the convective Froude number obtained from the numerical simulation. (b) The normalized rate compared with the Langley experimental curve reported by Pantano \& Sarkar (2002) that correlates the gas dynamic data with the convective Mach number.

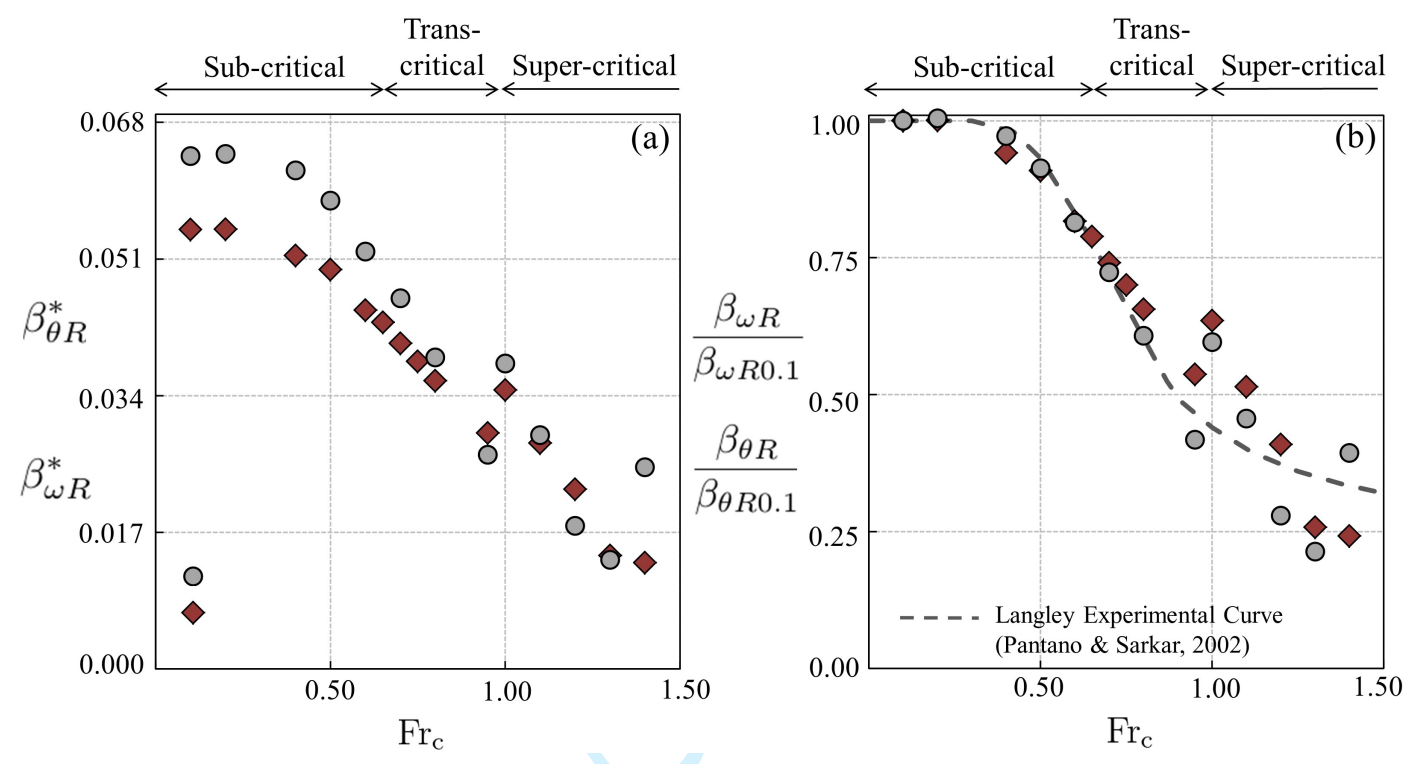

Published by NRC Research Press 
Fig. 7. Dependence of (a) the shape factor SF, (b) the radiation energy flux [II] ${ }^{*}$, and (c) the energy dissipation $\left([\mathrm{I}]^{*}-[\mathrm{II}]^{*}\right)$ on the convective Froude number $\mathrm{Fr}_{c}$. Solid-circle symbols denote the results obtained at the R-instant of time $t=t_{R}$. Open-circle symbols denote the results obtained at the $\mathrm{S}$-instant of time $t=t_{S}$. The ranges of the convective Froude number are: $\mathrm{Fr}_{c}<0.65$ for the sub-critical flow, $\mathrm{Fr}_{c}=0.65$ to 1.0 for the trans-critical flow, and $\mathrm{Fr}_{c}>1.0$ for the super-critical flow.

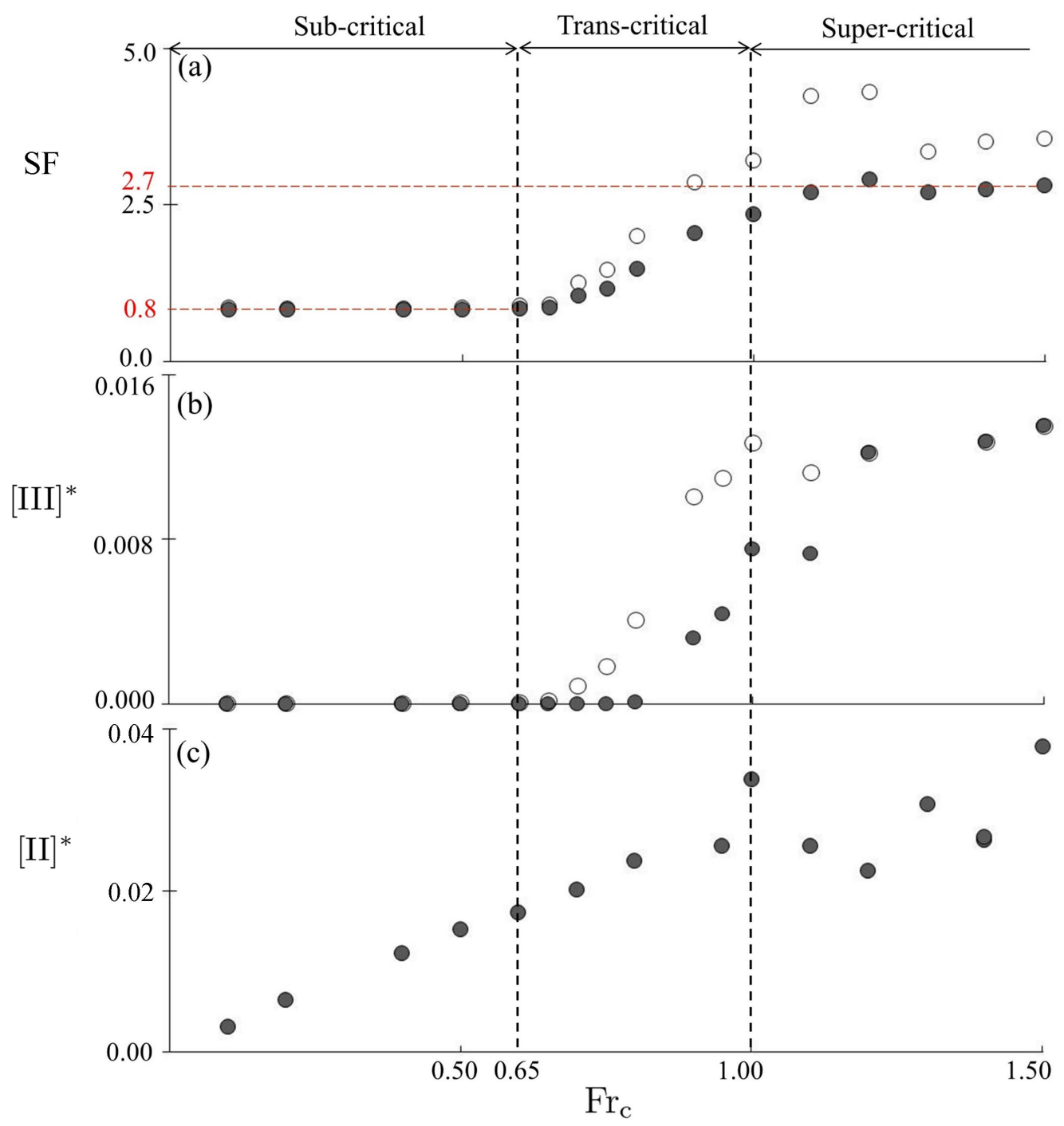


Fig. 8. The energy flux [II $]^{*}$ (first row) and the energy dissipation $[\mathrm{III}]^{*}$ (second row) obtained at the edges of two interrogation zones: (a) the sub-critical flow with $\mathrm{Fr}_{\mathrm{c}}=0.1$ and $k_{x o}^{*}=0.89$, (b) the trans-critical flow with $\operatorname{Fr}_{\mathrm{c}}=0.8$ and $k_{x o}^{*}=0.51$, and (c) the super-critical flow with $\operatorname{Fr}_{\mathrm{c}}=1.4$ and $k_{x o}^{*}=0.35$. The black lines denote the profiles obtained at the edges $y^{+}=-y^{-}=4 \delta_{\omega o}$. The red lines denote the profiles obtained at the edges $y^{+}=-y^{-}=12 \delta_{\omega o}$.

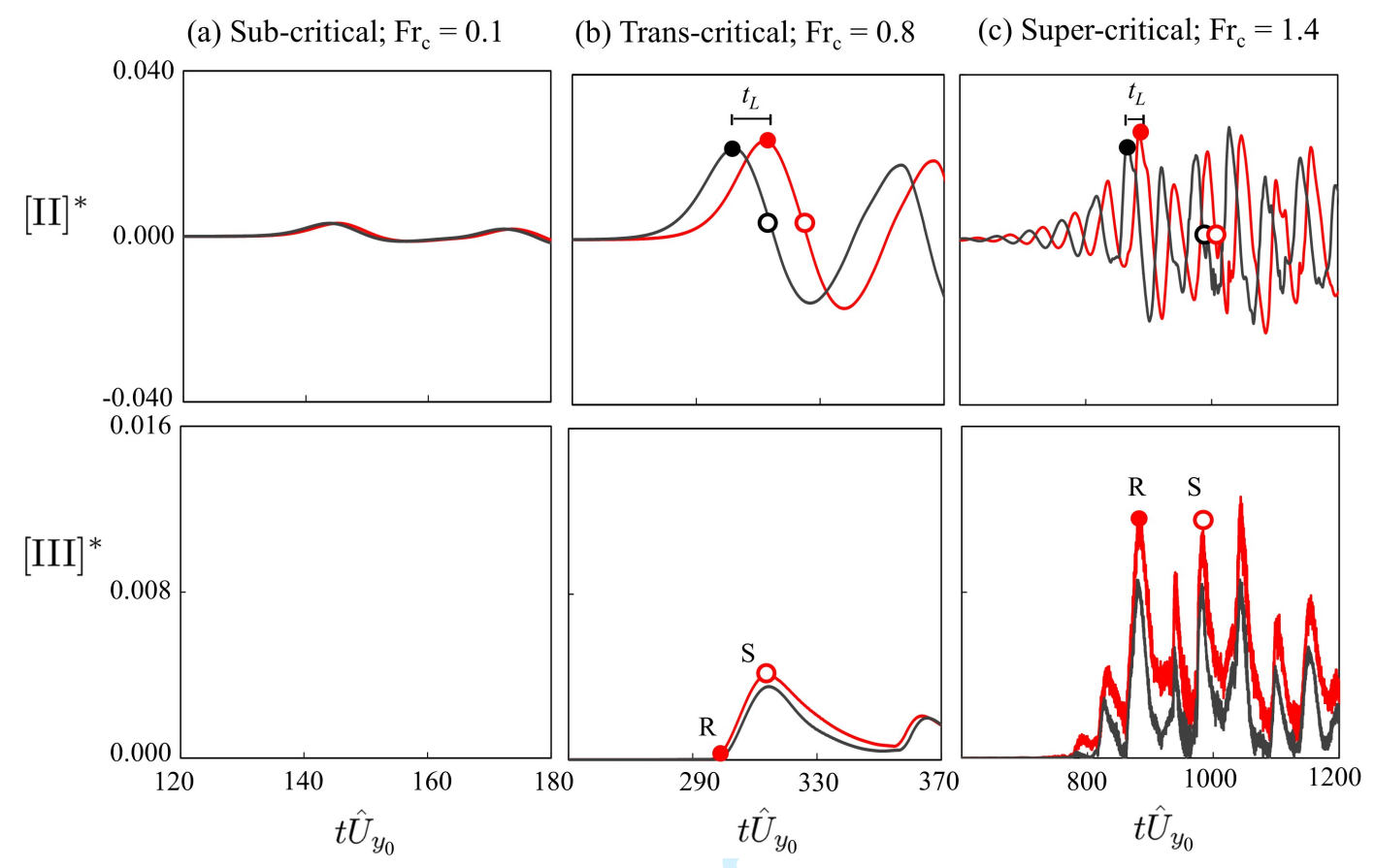




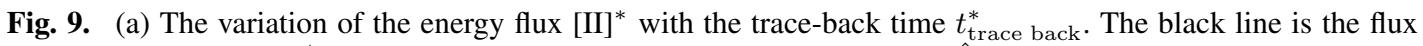
evaluated at the edges $y^{+}=-y^{-}=4 \delta_{\omega o}$ where $t_{\text {trace back }}^{*}=\left(t-4 \delta_{\omega o} / c\right) \hat{U}_{y o}$. The red line is the flux evaluated at the edges $y^{+}=-y^{-}=12 \delta_{\omega o}$ where $t_{\text {trace back }}^{*}=\left(t-12 \delta_{\omega o} / c\right) \hat{U}_{y o}$. (b) The variation of the momentum thickness $\delta_{\theta}^{*}$ with the time $t^{*}$. The solid-circle symbol marks the flux and the momentum thickness obtained at the R-instant of time $t^{*}=t_{R}^{*}$. The open-circle symbol marks the flux and the momentum thickness obtained at the S-instant of time $t^{*}=t_{S}^{*}$.

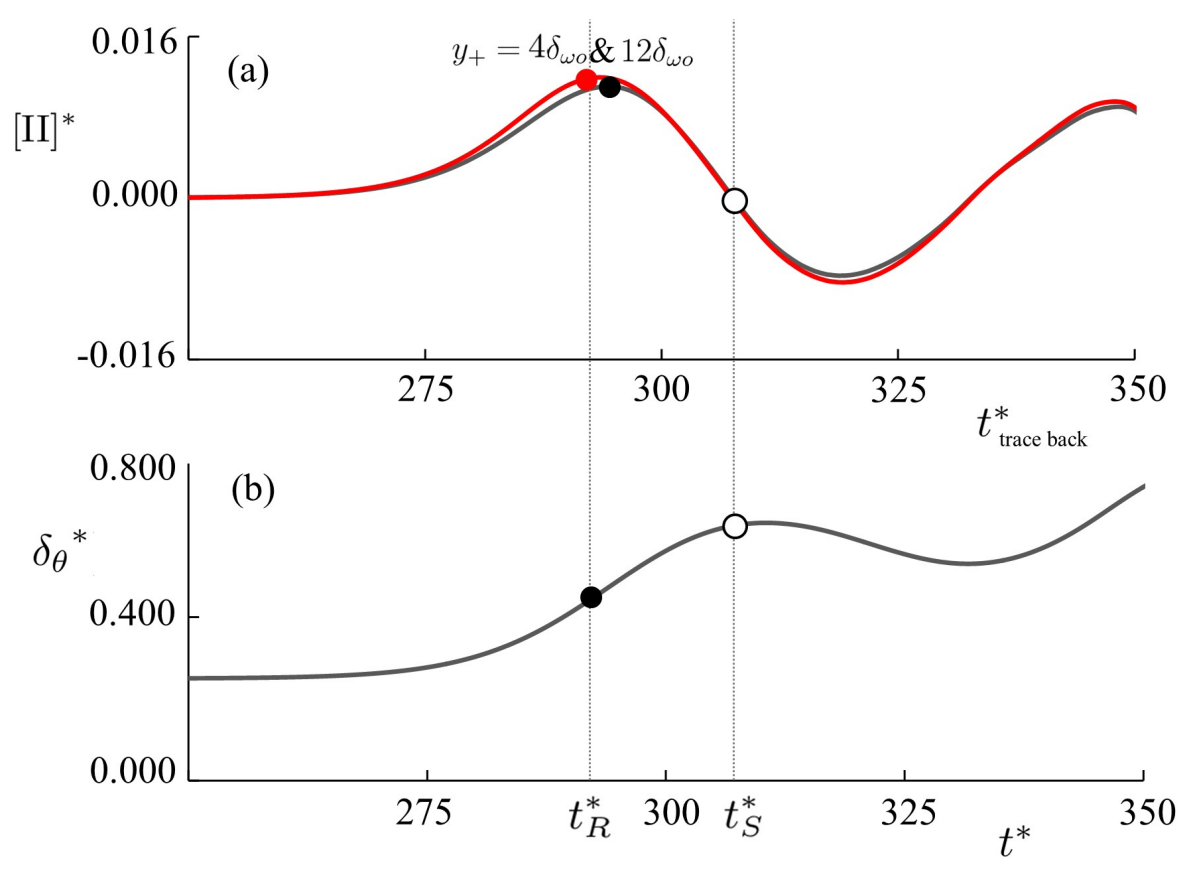

